\title{
iTRAQ-based quantitative proteomic analyses on the gender-specific responses in mussel Mytilus galloprovincialis to tetrabromobisphenol A
}

\author{
Chenglong $\mathrm{Ji}^{\mathrm{a}}$, Huifeng $\mathrm{Wu}^{\mathrm{a}, *}$, Lei Wei ${ }^{\mathrm{a}, \mathrm{b}}$, Jianmin $\mathrm{Zhao}^{\mathrm{a}}$ \\ ${ }^{a}$ Key Laboratory of Coastal Zone Environmental Processes, Yantai Institute of Coastal Zone Research (YIC), Chinese Academy of Sciences (CAS); Shandong \\ Provincial Key Laboratory of Coastal Zone Environmental Processes, YICCAS, Yantai 264003, P. R. China \\ ${ }^{\mathrm{b}}$ The University of Chinese Academy of Sciences, Beijing 100049, P. R. China
}

\section{A R T I C L E I N F O}

\section{Article history:}

Received 28 August 2014

Received in revised form

22 September 2014

Accepted 23 September 2014

Available online 30 September 2014

\section{Keywords:}

Tetrabromobisphenol A

Toxicological effects

Mytilus galloprovincialis

ITRAQ

Proteomics

\begin{abstract}
A B S T R A C T
Tetrabromobisphenol A (TBBPA) accounts for the largest production of brominated flame-retardants (BFRs) along the Laizhou Bay in China and is the most widely used BFR in industrial products. It can induce diverse toxicities including hepatotoxicity, nephrotoxicity, neurotoxicity and endocrine disrupting effects in mammalian and fish models. In this work, we applied iTRAQ-based proteomics to investigate the gender-specific responses in mussel Mytilus galloprovincialis to TBBPA. Thirty-one proteins were differentially expressed in hepatopancreas between male and female mussels, which clearly indicated the biological differences between male and female mussels at the protein level. After exposure of TBBPA $(18.4 \mathrm{nmol} / \mathrm{L})$ for one month, a total of 60 proteins were differentially expressed in response to the TBBPA treatment in mussel hepatopancreas, among which 33 and 29 proteins were significantly altered in TBBPA-treated male and female mussel samples, respectively. Only two of the 60 proteins were commonly altered in both male and female mussel samples exposed to TBBPA. Based on KEGG analysis, these differentially expressed proteins of TBBPA-induced effects were assigned to several groups, including cytoskeleton, reproduction and development, metabolism, signal transduction, gene expression, stress response and apoptosis. Overall, results indicated that TBBPA exposure could induce apoptosis, oxidative and immune stresses and disruption in energy, protein and lipid metabolisms in both male and female mussels with different mechanisms. This work suggested that the gender differences should be considered in ecotoxicoproteomics.
\end{abstract}

(C) 2014 Elsevier B.V. All rights reserved.

\section{Introduction}

Brominated flame retardants (BFRs) have been extensively used in a variety of industrial products, such as plastics, electronic equipment, textiles and upholstery foam, to reduce the risk of fire (de Wit, 2002). Among the BFRs, tetrabromobisphenol A (TBBPA) is nowadays the most frequently used BFR and can be released into marine environment (de Wit, 2002). Like other BFRs, TBBPA is a lipophilic substance and has a high persistency in the environment and therefore is globally traceable in multiple environmental samples including sediments and soils, marine and fresh water animals (Pan et al., 2010). Due to the abundant resources of seawater and underground brine, there are many industrial manufacturing plants of BFRs along the Laizhou Bay (Fig. S1) (Pan et al., 2010). The

\footnotetext{
* Corresponding author. Tel.: +86 535 2109190; fax: +86 5352109000.

E-mail address: hfwu@yic.ac.cn (H.Wu).
}

biggest manufacturing base for BFRs in China has been constructed and located in Weifang Binhai Economic Development Zone along the south coast of the Laizhou Bay (Pan et al., 2010). Zhang et al. (2011) reported that the TBBPA concentration in Mihe estuary was up to $1.24 \mathrm{nmol} / \mathrm{L}$, which contributed directly to the TBBPA pollution in the Laizhou Bay. Additionally, the released TBBPA can be potentially bioaccumulated by animals via the food chain in the aquatic environment (Morris et al., 2004). Therefore, the increasing release of TBBPA has attracted great attention worldwide because of its environmental persistence and potential toxicity to organisms (Johnson-Restrepoa et al., 2008).

TBBPA has diverse toxicities including hepatotoxicity, nephrotoxicity, neurotoxicity and cytotoxicity, which have been well documented (Szymanska et al., 1999; Birnbaum and Staskal, 2004; Strack et al., 2007). Szymanska et al. (1999) reported the hepatotoxicity of TBBPA in rat liver, indicated by the significant changes of biochemical indicators including glutathione (GSH), malondialdehyde (MDA) and 5-aminolevulinate (ALA-D). In addition, the 
endocrine disrupting effects induced by TBBPA were also found in different models including mammalian and fish (Meerts et al., 2000; Huang et al., 2013). The tissue of hepatopancreas is an important digestive and immune organ in bivalve functioning as innate immunity and detoxification (Du et al., 2013). Traditional toxicological approaches usually consist of histological studies on the lesions and measurement of specific responses such as the antioxidant activities for the test of oxidative stress or specifically expressed genes and proteins to test for certain toxicities without high-throughput analyses (Ji et al., 2013). Since researchers often target a known class of toxicity-responsive molecules, there are few opportunities to discover new molecules related to toxicological effects. These researches obviously presented a primary and less comprehensive understanding of toxicological responses of organisms to contaminants.

Mussel Mytilus galloprovincialis is frequently used as a good bioindicator in marine ecotoxicology because of its high tolerance and accumulation of contaminants (Ciacci et al., 2012). In addition, M. galloprovincialis can be easily sampled along the Bohai coast where is of the biggest manufacturing base of TBBPA in China. Since TBBPA is a known endocrine disrupting chemical to animals, the gender-specific effects of TBBPA should be considered in ecotoxicology, which may better understand the responsive mechanisms in toxicology bioindicators towards TBBPA exposure. To our knowledge, no studies attempted to compare the gender-specific responses induced by TBBPA in marine mussel at protein level. In this study, we used iTRAQ coupled with two-dimensional liquid chromatography-tandem mass spectrometry to characterize the differential proteomic changes and elucidate the differential toxicological effects of TBBPA in male and female mussels.

\section{Materials and methods}

\subsection{Animals}

Adult mussels M. galloprovincialis (shell length: $5.5-6.0 \mathrm{~cm}$, $n=30$ ) were collected in July 2012 in a pristine site (Yantai, China). After transported to the laboratory, the animals were acclimatized in aerated natural seawater (salinity $31 \mathrm{psu}$ ) for $7 \mathrm{~d}$ and were then divided into two groups (solvent control and treatment) each containing 15 mussels to ensure at least six male and six female individuals in each group. Dimethyl sulfoxide (DMSO) was used as the solvent for the dissolution of TBBPA (Guoyao, Shanghai, China). The mussel group cultured in the normal filtered seawater (FSW) containing $0.002 \%$ DMSO (v/v) were used as solvent control group. Since our previous experiment confirmed that there was no significant proteomic difference in mussel $M$. galloprovincialis samples between seawater control (mussels cultured in the FSW) and solvent (DMSO) control groups (Ji et al., 2013), we did not set the seawater control group in this study. The mussels in the treatment group were exposed to one sublethal concentration $(18.4 \mathrm{nmol} / \mathrm{L})$ of TBBPA. The concentration of TBBPA stock solution was $9.20 \mathrm{mmol} / \mathrm{L}$ in DMSO, ensuring the same DMSO concentration $(0.002 \%, v / v)$ in the TBBPA-exposed group to that of solvent control group. During the acclimation and exposure periods, mussels were kept at $25^{\circ} \mathrm{C}$ under a photoperiod of $12 \mathrm{~h}$ light and $12 \mathrm{~h}$ dark, and fed with the Chlorella vulgaris at a ration of $2 \%$ of tissue per dry weight daily. After exposure for 1 month, all the mussels were immediately dissected for hepatopancreas and gonad tissues. The gonad tissues were used for sex determination. Each hepatopancreas tissue sample was divided into three parts that were used for histological observation, TUNEL assay and protein and RNA extraction. Those hepatopancreas samples for protein and RNA extraction were snap-frozen in liquid nitrogen, and then stored at $-80^{\circ} \mathrm{C}$. For iTRAQ-based proteomics, either solvent control group or TBBPA-exposed group consisted of two (three pooled into one) biological replicates for both male and female mussels, respectively.

\subsection{Histology}

Both gonad and hepatopancreas tissues were carefully fixed in the Bouin's fixative solution after dissection from the mussels. The histology of gonad tissues was simply used for sex determination (Fig. S2). After fixation for $24 \mathrm{~h}$, the tissues were then dehydrated in a progressive series of ethanol and embedded in paraffin. Tissues from control and TBBPA treatment $(18.4 \mathrm{nmol} / \mathrm{L})$ were processed together in batches to remove artefacts between control and TBBPA treatment. Histological sections ( $6-8 \mu \mathrm{m}$ thickness) were cut from the paraffin embedded tissues and mounted on slides which were stained with hematoxylin-eosine (HE) and observed under a light microscope (Olympus BX61, Tokyo, Japan) at $\times 200$ magnification (zoom on the camera was $\times 2.5$ ). For each individual animal, there were three sections examined. The histological observations were randomly made on five fields of one section per sample. A mean for each section was derived by randomly counting at least 10 tubules.

\subsection{TUNEL assay}

Terminal dUTP nick-end labeling technique (TUNEL-technique) was applied to detect the apoptosis in hepatopancreas and performed with one step TUNEL kit (Beyotime Institute of Biotechnology, Haimen, Jiangsu, China) according to the manufacturer's instructions. More details about TUNEL technique were described in the Supporting Information.

\subsection{Quantitative proteomic analysis}

Each pooled hepatopancreas sample was ground into powder in liquid nitrogen and then dissolved in lysis buffer ( $9 \mathrm{M}$ urea, $4 \%$ CHAPS, $1 \% \mathrm{w} / \mathrm{v}$ DTT and $1 \%$ IPG buffer) with protease inhibitor (Roche Applied Science, Mannheim, Germany) to extract proteins. The concentrations of the protein extracts were determined using the Bradford method (Bradford, 1976).iTRAQ technique was applied to quantitative proteomic analysis. The iTRAQ labeling of peptides from mussel samples of solvent control and TBBPA exposure were performed using iTRAQ 8-plex reagents (Applied Biosystems, Foster City, CA, USA) according to the manufacturer's protocol. Eight samples (two biological replicates per group) were labeled with the iTRAQ tags as female control, female TBBPA treatment, male control and male TBBPA treatment tags). Protein Pilot Software 4.0 (AB SCIEX, Framingham, MA, USA) was used to process proteomic data against a marine bivalve-EST-Translated protein database (Mytilus galloprovincialis, Mytilus edulis, Ruditapes philippinarum and Crassostrea gigas, 238224 sequences) using the Paragon algorithm (Shilov et al., 2007). The protein ratios in each replicate were then quantified based on the summed intensities of the matched spectrum. These ratios from the biological replicates were evaluated by using a Student's $t$-test combined with the Benjamini-Hochberg correction (Han et al., 2013). Proteins with corrected $p$ values less than 0.05 and fold changes larger than 1.20 or smaller than 0.83 were considered to be significantly differential. More details about quantitative proteomic analysis were described in the Supporting Information.

\subsection{RNA extraction and $q R T-P C R$}

In order to evaluate the correlation between mRNA expression and protein abundances, qRT-PCR was used to determine the expression levels of mRNA. More details of RNA extraction and qRT-PCR were described in the Supporting Information. 

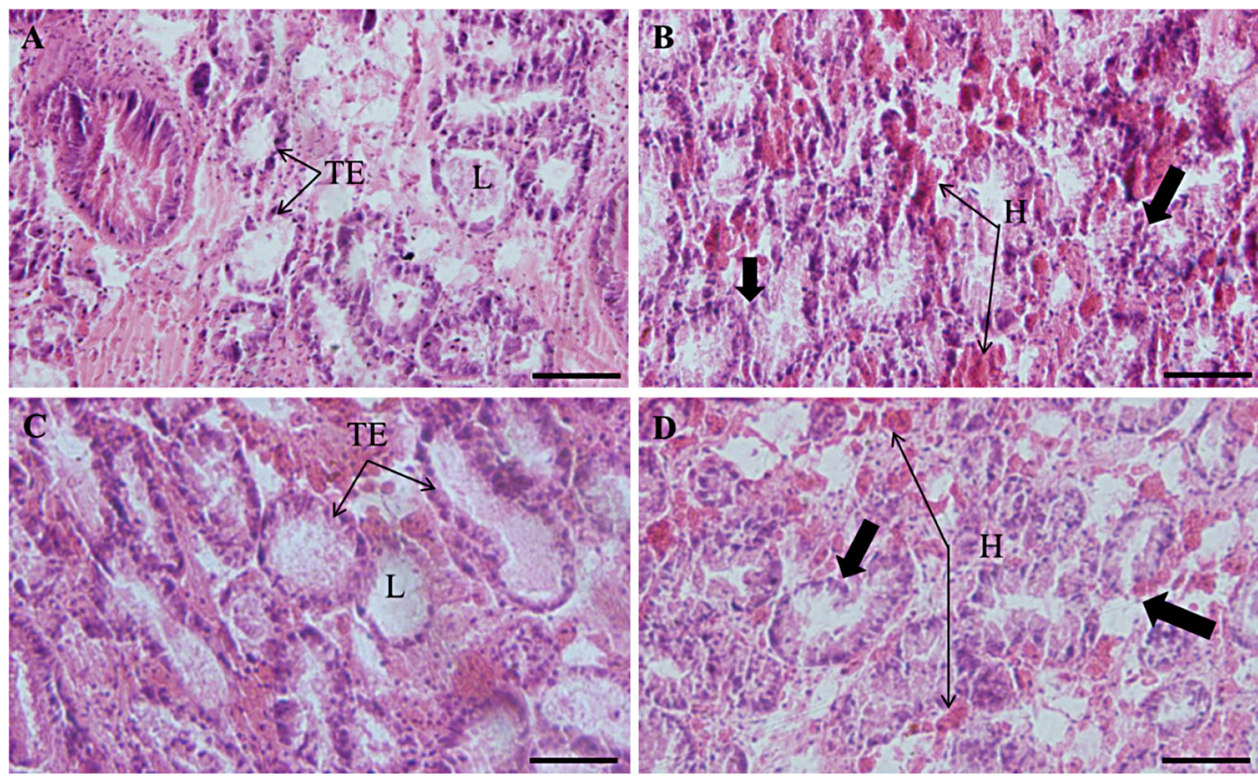

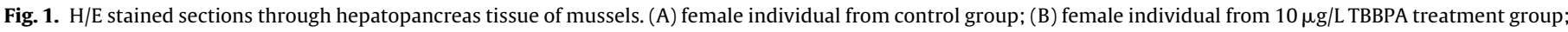

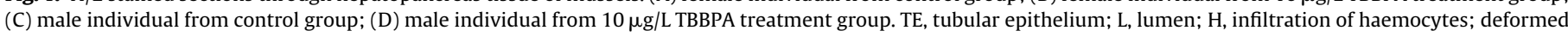
tubules (bold arrow). Scale bar: $40 \mu \mathrm{m}$.

\section{Results}

\subsection{Histological observation of hepatopancreas from mussels exposed to TBBPA}

Sections of hepatopancreas from control and TBBPA-exposed mussels are exhibited in Fig. 1. No significant histological differences were observed between female control (Fig. 1A) and male control (Fig. 1C) hepatopancreas samples presenting normal architecture of hepatopancreatic tubule and lumen. In contrast, the hepatopancreas tissues showed infiltration of haemocytes as well as deformation of tubules in TBBPA-exposed female mussels (Fig. 1B). The similar appearances were also observed in hepatopancreas from male mussel samples with TBBPA exposure (Fig. 1D).

\subsection{Apoptosis in the hepatopancreas from mussels exposed to TBBPA}

TUNEL technique was performed on hepatopancreas tissue sections to assess the occurrences of apoptotic nuclei in individual cells. In the control groups, as shown in Fig. 2A andC, no obvious apoptotic nuclei were found in either epithelial cells or interstitial connective tissue (ICT) in both male and female mussel hepatopancreas tissues from control groups. In TBBPA treatment group, epithelial cells apoptosis was not found in either female or male mussel samples. However, the ICT of hepatopancreas from both male and female TBBPA treatment groups showed significant higher levels of apoptotic nuclei than those from control groups (Fig. 2B and D), which meant the occurrences of apoptosis induced by TBBPA exposure in ICT of hepatopancreas from both male and female TBBPA treatment groups. The apoptosis appearances did not demonstrate gender-specific responses to TBBPA treatment in mussel hepatopancreas tissues.

\subsection{Proteomic differences in hepatopancreas between male and female mussels}

In total, 1043 proteins were quantified on the basis of 23376 highly confident spectra, of which 10231 peptides were unique. On average, each protein was quantified using 22.4 spectra. Among the 1043 proteins, 31 proteins showed significantly $(p<0.05)$ different abundances in hepatopancreas between male and female mussels from control group (Table 1 ), including 15 and 16 abundant proteins in male and female mussel samples, respectively. Pathways of these differential proteins were analyzed using the KEGG database. According to the KEGG pathway analysis, these proteins were basically involved in cytoskeleton, reproduction and development, energy and primary metabolism, translation, stress response and signal transduction.

\subsection{Differential proteomic responses in hepatopancreas between male and female mussels exposed to TBBPA}

Comparison between control and TBBPA-exposed mussel groups indicated that 60 proteins were significantly $(p<0.05)$ changed in expression from both male and female mussel samples. A total of 33 proteins displayed significant changes in expression, in which 8 proteins showed increased expression, while 25 showed decreased expression in male mussel samples exposed to TBBPA for one month (Table 2 and Fig. 3). Among these 33 significantly altered proteins in male TBBPA-treated mussel group, only three (9.1\%) of them were also found to be of significant differences between male control and female control groups. For the female mussel samples, there were 29 proteins altered in TBBPA-treated group, including 19 up-regulated and 10 down-regulated proteins. Interestingly, twelve proteins (41.4\%) among these 29 proteins in response to TBBPA exposure were gender-specific in female mussel samples with significantly different abundances compared to those in male mussel samples (Fig. 3). However, only two proteins (3.3\%) were commonly changed in expression from both male and female mussel samples exposed to TBBPA. Fig. 4 summarized the pathways related to the proteomic response of $M$. galloprovincialis to the TBBPA treatment.

\subsection{Correlation between gene expressions and protein abundances}

To further verify the results of protein expressions and compare the correlation between protein and gene expressions, seven representative genes related to altered proteins, including 

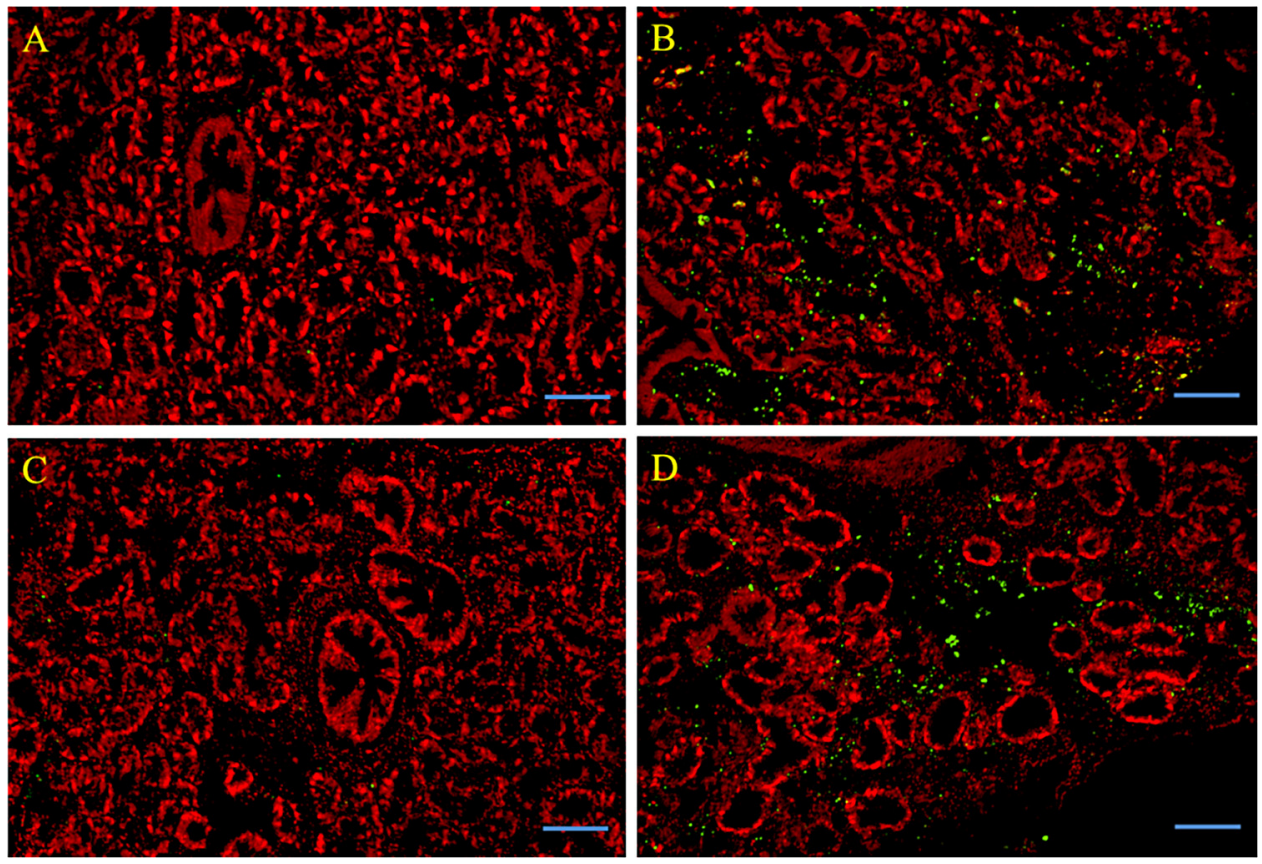

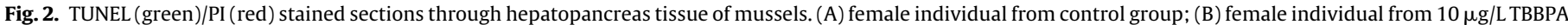
treatment group; (C) male individual from control group; (D) male individual from $10 \mu \mathrm{g} / \mathrm{L}$ TBBPA treatment group. Scale bar: $50 \mu \mathrm{m}$.

Table 1

Differentially expressed proteins between male and female mussel groups.

\begin{tabular}{|c|c|c|c|c|}
\hline Accession & Description & No. of unique peptides & $p$ value $^{a}$ & $\begin{array}{l}\text { Ratio } \\
\text { (male/female) }\end{array}$ \\
\hline \multicolumn{5}{|c|}{ Cytoskeleton } \\
\hline FL499829 & $\beta$-actin & 134 & 0.0136 & 5.10 \\
\hline FL494680 & Suprabasin & 2 & 0.0232 & 0.82 \\
\hline FL489343 & RecName: Tropomyosin >gb|AAA82259.1| & 40 & 0.0444 & 0.59 \\
\hline FL489336 & Cytoplasmic dynein 1 light intermediate chain 1 & 1 & 0.0389 & 1.39 \\
\hline AJ625699 & Tropomyosin >dbj|BAA19209.1| & 31 & 0.0238 & 0.48 \\
\hline \multicolumn{5}{|c|}{ Reproduction and development } \\
\hline FL594305 & Protamine-like PL-II/PL-IV precursor & 2 & 0.0359 & 2.13 \\
\hline FL493691 & Meiosis-specific nuclear structural protein 1 & 5 & 0.0030 & 8.24 \\
\hline FL493456 & Yolk ferritin & 4 & 0.0470 & 0.44 \\
\hline FL491285 & Zonadhesin & 3 & 0.0034 & 4.09 \\
\hline AJ625943 & Putative vitelline envelop receptor for lysin & 1 & 0.0310 & 0.29 \\
\hline \multicolumn{5}{|l|}{ Metabolism } \\
\hline FL497290 & PREDICTED: L-xylulose reductase-like isoform X1 & 10 & 0.0228 & 0.47 \\
\hline FL496349 & Glyceraldehyde-3-phosphate dehydrogenase & 56 & 0.0291 & 13.68 \\
\hline FL494162 & Glycosyl hydrolase & 2 & 0.0347 & 13.30 \\
\hline FL492895 & Low-density lipoprotein receptor-related protein 6 & 5 & 0.0338 & 0.23 \\
\hline FL489629 & Arginine kinase & 33 & 0.0015 & 6.61 \\
\hline AJ625434 & PREDICTED: glucose dehydrogenase [acceptor]-like & 14 & 0.0440 & 0.47 \\
\hline AJ624848 & Pyruvate carboxylase, mitochondrial & 1 & 0.0031 & 1.68 \\
\hline \multicolumn{5}{|c|}{ Signal transduction } \\
\hline FL493105 & Calponin-like protein & 14 & 0.0078 & 2.01 \\
\hline AJ624341 & Putative epidermal cell surface receptor & 2 & 0.0194 & 3.60 \\
\hline AJ623579 & Sarcoplasmic calcium-binding protein & 14 & 0.0171 & 0.58 \\
\hline \multicolumn{5}{|l|}{ Translation } \\
\hline FL495584 & Ribosomal protein $\mathrm{L}$ & 5 & 0.0406 & 0.72 \\
\hline AM879598 & Ribosomal protein L19 & 6 & 0.0344 & 0.73 \\
\hline FL492189 & Ribosomal protein L9 & 9 & 0.0221 & 0.72 \\
\hline \multicolumn{5}{|c|}{ Stress response } \\
\hline FL499875 & 78 kDa glucose-regulated protein, partial & 63 & 0.0367 & 0.44 \\
\hline FL496423 & Apextrin-like protein & 5 & 0.0308 & 10.96 \\
\hline FL495401 & Chitotriosidase-1 & 104 & 0.0260 & 0.74 \\
\hline FL494057 & Glutathione S-transferase A & 9 & 0.0409 & 1.58 \\
\hline AJ626434 & Chitotriosidase & 133 & 0.0354 & 0.55 \\
\hline \multicolumn{5}{|l|}{ Others } \\
\hline FL497051 & Hypothetical protein CGI_10021496 & 13 & 0.0062 & 0.42 \\
\hline FL489129 & Hypothetical protein & 1 & 0.0036 & 1.25 \\
\hline AJ625534 & PREDICTED: uncharacterized protein LOC100179434 & 19 & 0.0027 & 5.60 \\
\hline
\end{tabular}

\footnotetext{
a $p$ values have been corrected using the Benjamini-Hochberg method.
} 
Table 2

Differentially expressed proteins in response to TBBPA treatment in male and female mussel hepatopancreas.

\begin{tabular}{|c|c|c|c|c|}
\hline Accession & Description & No. of unique peptides & $p$ value $^{a}$ & Fold change $^{\mathrm{b}}$ \\
\hline \multicolumn{5}{|c|}{ Cytoskeleton } \\
\hline FL594178 & Profilin & 34 & 0.0065 & $-3.13^{c}$ \\
\hline FL499932 & Kinesin-related protein 1 & 1 & 0.0343 & $-7.45^{\mathrm{c}}$ \\
\hline FL497207 & Tubulin A & 51 & 0.0029 & $-2.94^{\mathrm{c}}$ \\
\hline FL494680 & Suprabasin & 2 & 0.0122 & $1.64^{\mathrm{c}}$ \\
\hline AJ624420 & Actin & 112 & 0.0372 & $3.10^{c}$ \\
\hline FL500031 & Septin-2 & 2 & 0.0449 & $3.10^{\mathrm{d}}$ \\
\hline FL497199 & Non-neuronal cytoplasmic intermediate filament protein & 43 & 0.0322 & $2.09^{\mathrm{d}}$ \\
\hline FL492621 & Catchin protein & 9 & 0.0452 & $-2.07^{\mathrm{d}}$ \\
\hline AJ625699 & Tropomyosin >dbj|BAA19209.1| & 31 & 0.0495 & $-1.99^{\mathrm{d}}$ \\
\hline \multicolumn{5}{|c|}{ Reproduction and development } \\
\hline FL633554 & Vdg3 & 5 & 0.0498 & $-4.61^{\mathrm{c}}$ \\
\hline FL491285 & Zonadhesin & 3 & 0.0047 & $1.49^{\mathrm{d}}$ \\
\hline AJ625943 & Putative vitelline envelop receptor for lysin & 1 & 0.0297 & $-3.80^{\mathrm{d}}$ \\
\hline FL493691 & Meiosis-specific nuclear structural protein 1 & 5 & 0.0047 & $1.66^{\mathrm{d}}$ \\
\hline \multicolumn{5}{|l|}{ Metabolism } \\
\hline FL501230 & Procollagen-proline dioxygenase beta subunit & 18 & 0.0060 & $-4.29^{c}$ \\
\hline FL495257 & Ganglioside GM2 activator & 2 & 0.0493 & $-2.61^{\mathrm{c}}$ \\
\hline FL492537 & Proteasome subunit beta type- 1 & 3 & 0.0348 & $1.94^{\mathrm{c}}$ \\
\hline FL490476 & Isoamyl acetate-hydrolyzing esterase 1 -like protein & 1 & 0.0370 & $-5.35^{\mathrm{c}}$ \\
\hline FL490188 & Cob(I)yrinic acid a,c-diamide adenosyltransferase, mitochondrial-like & 1 & 0.0357 & $-1.20^{\mathrm{c}}$ \\
\hline AM879460 & Fatty acid-binding protein & 10 & 0.0295 & $-1.77^{c}$ \\
\hline AJ625020 & Electron transfer flavoprotein subunit alpha, mitochondrial-like isoform X1 & 7 & 0.0282 & $-1.61^{\mathrm{c}}$ \\
\hline FL594008 & ATP synthase beta subunit, partial & 46 & 0.0336 & $-3.50^{c}$ \\
\hline FL496349 & Glyceraldehyde-3-phosphate dehydrogenase & 56 & 0.0344 & $2.75^{\mathrm{d}}$ \\
\hline J625434 & Glucose dehydrogenase [acceptor]-like & 14 & 0.0265 & $2.07^{\mathrm{d}}$ \\
\hline FL489629 & Arginine kinase & 33 & 0.0017 & $4.88^{\mathrm{d}}$ \\
\hline \multicolumn{5}{|c|}{ Signal transduction } \\
\hline FL497430 & Collagen alpha-5(VI) chain & 3 & 0.0196 & $7.73^{c}$ \\
\hline \multirow[t]{2}{*}{ AJ626069 } & Collagen alpha-1(XII) chain & 87 & 0.0113 & $2.78^{\mathrm{c}}$ \\
\hline & & & 0.0001 & $1.80^{\mathrm{d}}$ \\
\hline FL494852 & Tyrosine-protein phosphatase non-receptor type 6 & 1 & 0.0275 & $-4.66^{c}$ \\
\hline AJ516340 & Calcium binding protein 1 & 18 & 0.0347 & $-2.09^{c}$ \\
\hline AJ624341 & Putative epidermal cell surface receptor & 2 & 0.0234 & $3.08^{\mathrm{d}}$ \\
\hline \multicolumn{5}{|c|}{ Gene expression } \\
\hline FL496796 & Elongation factor 1-gamma & 7 & 0.0288 & $-2.25^{c}$ \\
\hline EH663238 & Ribosomal protein & 16 & 0.0083 & $-6.49^{c}$ \\
\hline \multirow[t]{2}{*}{ AM878418 } & Nucleoprotein TPR & $1^{\mathrm{e}}$ & 0.0034 & $-1.26^{\mathrm{c}}$ \\
\hline & & & 0.0089 & $-2.54^{\mathrm{d}}$ \\
\hline AJ624092 & Excinuclease ABC subunit B & 138 & 0.0074 & $2.27^{c}$ \\
\hline FL496868 & Transcription activator & 3 & 0.0285 & $-5.20^{\mathrm{d}}$ \\
\hline FL495374 & Elongation factor 2 & 22 & 0.0216 & $3.08^{\mathrm{d}}$ \\
\hline FL492837 & $40 \mathrm{~S}$ ribosomal protein $\mathrm{S} 4, \mathrm{X}$ isoform & 8 & 0.0135 & $-2.28^{\mathrm{d}}$ \\
\hline FL489105 & PREDICTED: Serine/arginine-rich splicing factor 4-like isoform X1 & 3 & 0.0193 & $2.91^{\mathrm{d}}$ \\
\hline AM880346 & QM-like protein & 9 & 0.0326 & $2.01^{\mathrm{d}}$ \\
\hline AJ625362 & $60 S$ ribosomal protein L24-like & 5 & 0.0197 & $2.29^{\mathrm{d}}$ \\
\hline \multicolumn{5}{|c|}{ Stress response } \\
\hline FL595030 & Selenium-binding protein 1 , partial & 7 & 0.0128 & $-2.65^{\mathrm{c}}$ \\
\hline FL499875 & 78 kDa glucose-regulated protein, partial & 63 & 0.0007 & $-4.41^{\mathrm{c}}$ \\
\hline FL499581 & Peroxiredoxin 6 & 17 & 0.0296 & $-10.19^{c}$ \\
\hline FL499562 & Hsc70-interacting protein & 8 & 0.0022 & $-5.75^{c}$ \\
\hline FL491817 & Manganese superoxide dismutase & 8 & 0.0020 & $-5.06^{c}$ \\
\hline FL492107 & TCTP & 12 & 0.0343 & $-3.50^{c}$ \\
\hline FL593839 & Calreticulin & 35 & 0.0234 & $-3.28^{c}$ \\
\hline AJ625233 & Acidic mammalian chitinase isoform $\mathrm{X} 2$ & 43 & 0.0349 & $3.87^{c}$ \\
\hline AJ624097 & Chitotriosidase & 133 & 0.0293 & $1.36^{\mathrm{c}}$ \\
\hline FL492661 & Lectin & 20 & 0.0459 & $-2.94^{\mathrm{c}}$ \\
\hline FL496702 & Lysozyme & 7 & 0.0357 & $8.39^{\mathrm{d}}$ \\
\hline FL496423 & Apextrin-like protein & 5 & 0.0336 & $8.47^{\mathrm{d}}$ \\
\hline FL495401 & Chitotriosidase- 1 & 104 & 0.0002 & $2.18^{\mathrm{d}}$ \\
\hline \multicolumn{5}{|l|}{ Apoptosis } \\
\hline FL497051 & Apoptosis 2 inhibitor & 13 & 0.0036 & $-12.24^{\mathrm{d}}$ \\
\hline FL491544 & Cell death protein 3-like & 4 & 0.0247 & $-8.71^{\mathrm{d}}$ \\
\hline \multicolumn{5}{|l|}{ Others } \\
\hline FL489129 & Hypothetical protein & 1 & 0.0036 & $-1.25^{\mathrm{c}}$ \\
\hline FL497768 & Hypothetical protein CGI_10012958 & 15 & 0.0122 & $-1.44^{\mathrm{d}}$ \\
\hline FL494257 & Hypothetical protein CGI_10009690 & 23 & 0.0271 & $-4.09^{\mathrm{d}}$ \\
\hline FL494162 & Hypothetical protein & 2 & 0.0357 & $11.37^{\mathrm{d}}$ \\
\hline AJ625057 & Hypothetical protein EAG_11213 & 11 & 0.0221 & $2.85^{\mathrm{d}}$ \\
\hline AJ625534 & PREDICTED: uncharacterized protein LOC100179434 & 19 & 0.0023 & $3.41^{\mathrm{d}}$ \\
\hline
\end{tabular}

a $p$ values have been corrected using the Benjamini-Hochberg method.

b Plus (+) which is omitted represents up-regulated expression, and minus (-) represents down-regulated expression.

c Altered proteins in male mussels.

d Altered proteins female mussels. 


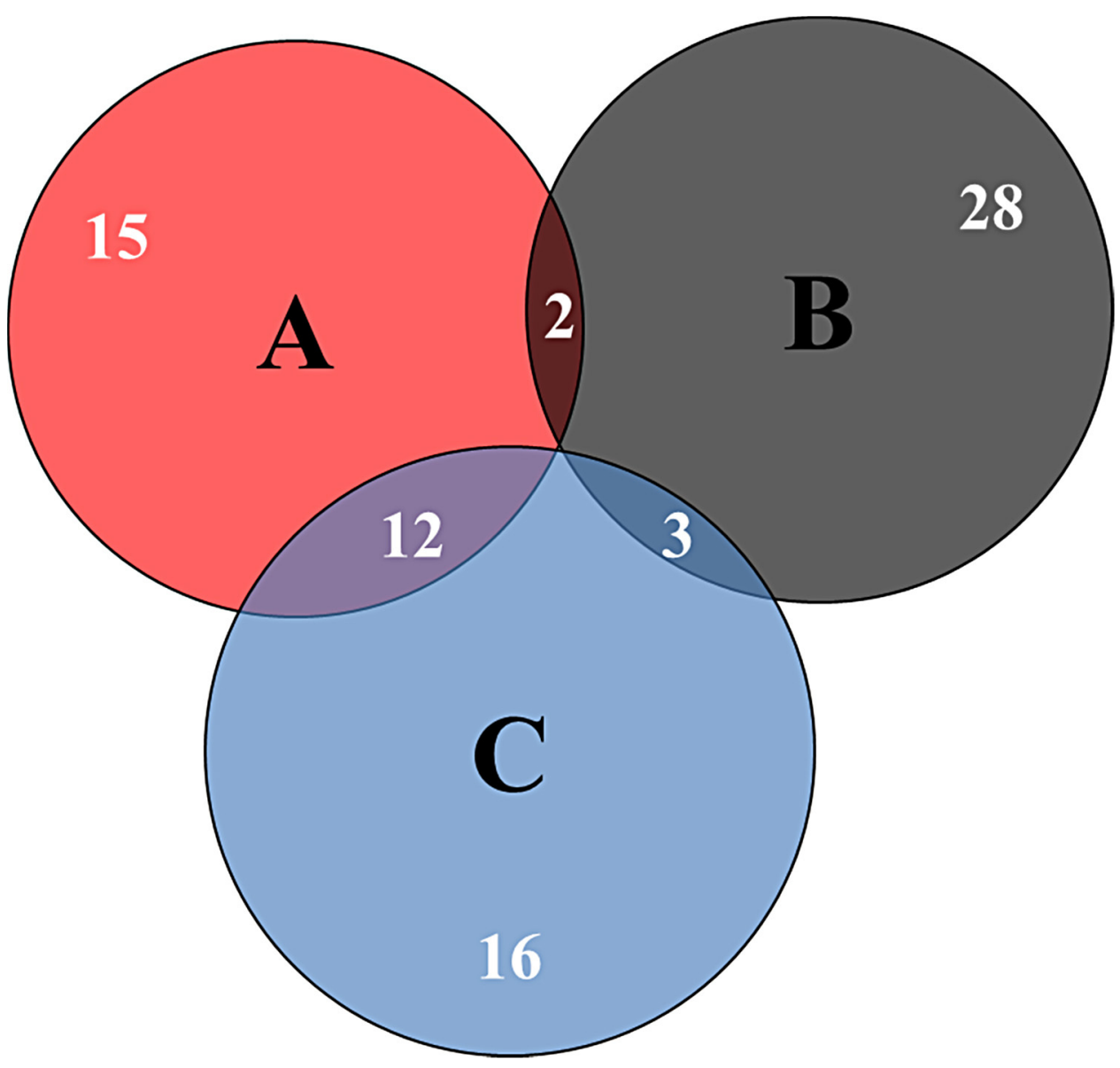

Fig. 3. A Venn diagram showing the overlaps between TBBPA-related proteins from females (A) and males (B), and gender-specific proteins (C).

nucleoprotein translocated promoter region protein (TPR) and $78 \mathrm{kDa}$ glucose-regulated protein (GRP78) in male group and TPR, glucose dehydrogenase [acceptor]-like (GDA), epidermal cell surface receptor (ECSR), arginine kinase (AK), apextrin-like protein (ALP) and apoptosis 2 inhibitor (A2I) in female group, were quantified using qRT-PCR. The results indicated that the mRNA expression levels of both TPR and GRP78 had consistent alteration tendency with corresponding proteins in male TBBPA-treatment group. In female TBBPA-treatment group, TPR, GDA, ECSR and A2I demonstrated positive correlation between mRNA and protein expressions, while AK and ALP presented disparity between mRNA expression and corresponding protein abundance (Fig. 5).

\section{Discussion}

With the rapid development of -omic techniques including genomics, transcriptomics, proteomics and metabolomics, researchers can focus on a global profile of one type of molecules such as genes, proteins and metabolites and their alterations with high-throughput analyses, which offer great potential in unraveling toxicological effects and mechanisms of environmental contaminants (Weckwerth, 2011; Fasulo et al., 2012; Cappello et al., 2013a,b; Ji et al., 2013). Among these approaches, proteomics is a large-scale study of proteins encoded by the given genome in an organism (Anderson and Anderson, 1998). Not only can proteomics describe complete proteomes at tissue, cell, or organelle levels, but it can also be used to compare proteomes under environmental contaminant stresses (Ahsan et al., 2009). iTRAQ is a gel-free technique used to quantify proteins from different samples in a single experiment by using isotope-coded covalent tags (Pütz et al., 2012; Han et al., 2013) and has been popularly employed in quantitative proteomic studies due to its relatively high sensitivity and reproducibility (Martyniuk et al., 2012; Han et al., 2013). In this study, we explored the gender-specific response in hepatopancreas of mussel M. galloprovincialis to TBBPA by using the iTRAQ-based proteomics.

\subsection{TBBPA induced damages and apoptosis in mussel hepatopancreas}

Histological observations may provide straightforward evidences on the tissue disorders related to the status of organisms. In both male and female samples with the TBBPA treatment, the hepatopancreas displayed conspicuous haemocytic infiltration and severe lesions of tubules. Infiltration of haemocytes in response to exposure to organic pollutants, such as PAHs and a mixture of diesel oil, has been reported in marine mussels (Auffert, 1988; Cappello et al., 2013a,b) and was interpreted as a repair process following tissue damage (Garmendia et al., 2011). Moore (1985) assumed that autolytic process of the epithelia of hepatopancreas tubule was a consequence of full lysosomal destabilization, which was confirmed by Auffert (1988) who found the incidence of lysosomes was inversely correlated to the extent of tubule lesions.

TUNEL technique was used to assess apoptosis of hepatopancreas from TBBPA-exposed mussels. Results indicated that ICT represented the distinct apoptotic nuclei. As a part of hepatopancreas, ICT surrounding the digestive tubules plays an important role in fixing and protecting tubules. Minguez et al. (2013) found that zebra mussels infected by bacterium showed apoptotic nuclei 


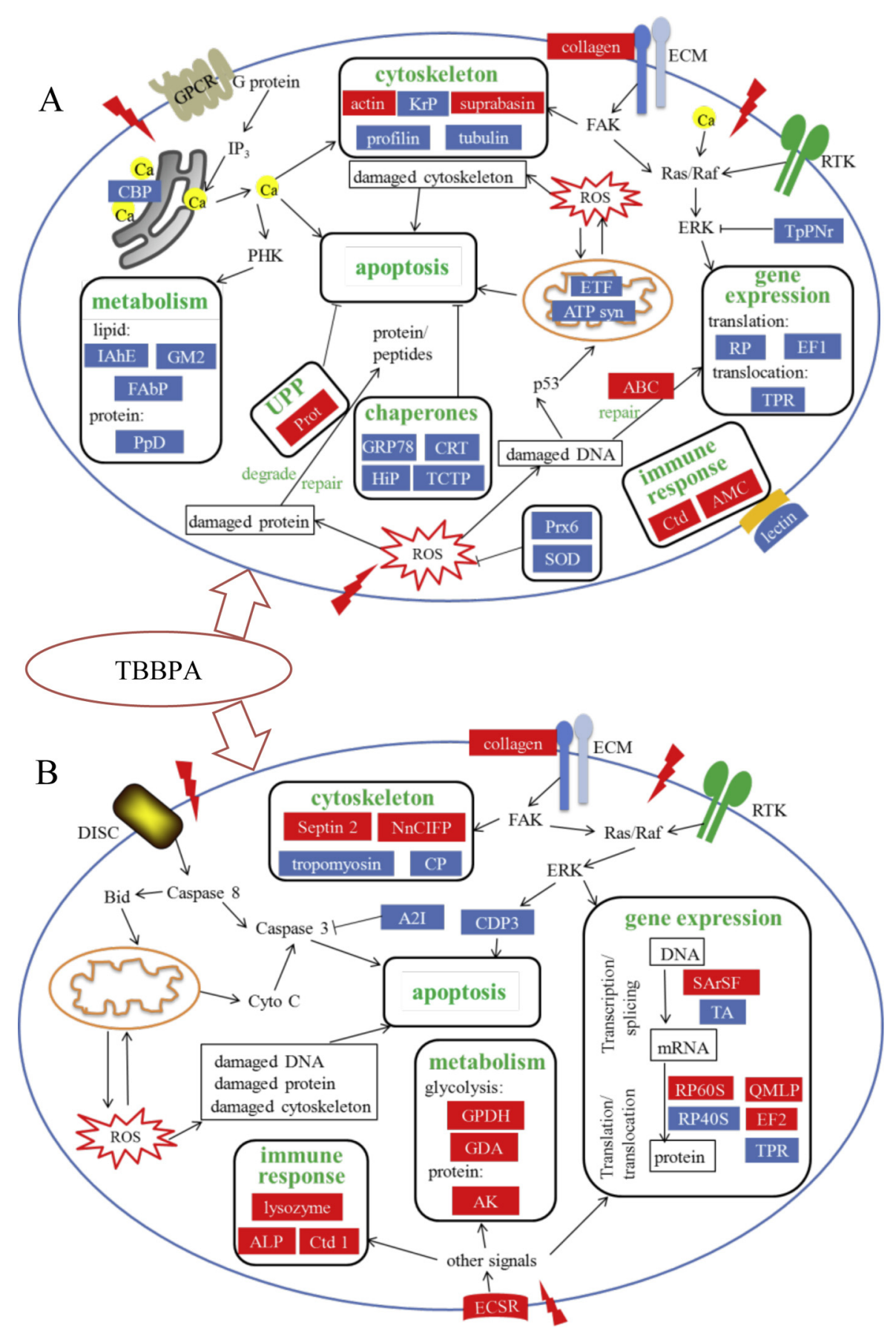

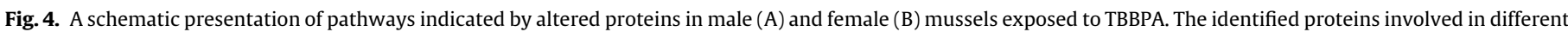

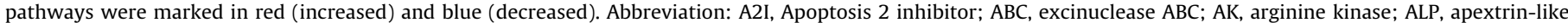

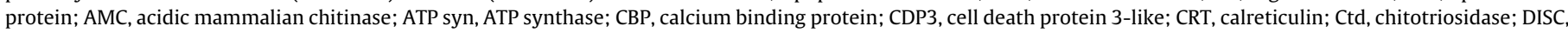

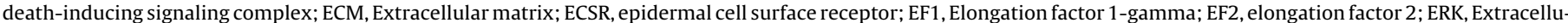

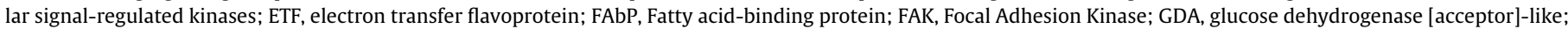

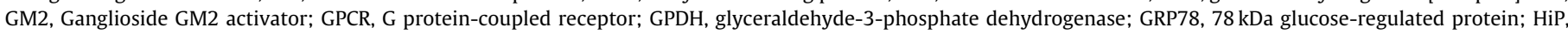

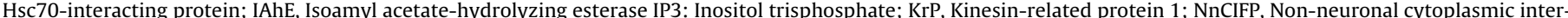

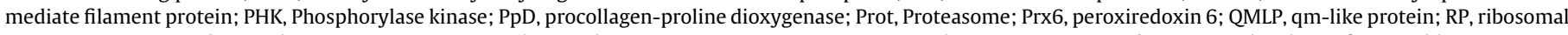

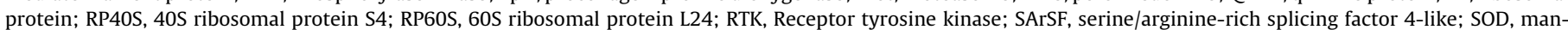

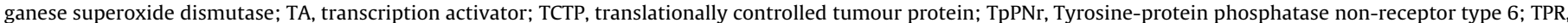
Nucleoprotein translocated promoter region protein; UPP, ubiquitin-proteasome pathway.

near the digestive gland, whereas the digestive tubules showed no stained cells, which was similar to TBBPA-induced apoptosis in hepatopancreas in marine mussels in this study.

Both histological observations and TUNEL assay indicated that TBBPA treatment could induce clear haemocytic infiltration, lesions of tubules and apoptosis in mussel hepatopancreas. However, these results did not present potentially differential responses between male and female mussel samples towards TBBPA exposure. To elucidate the gender-specific responses and molecular mechanisms, an iTRAQ-based proteomic approach was used to quantitatively profile $M$. galloprovincialis proteins in response to TBBPA treatment. 

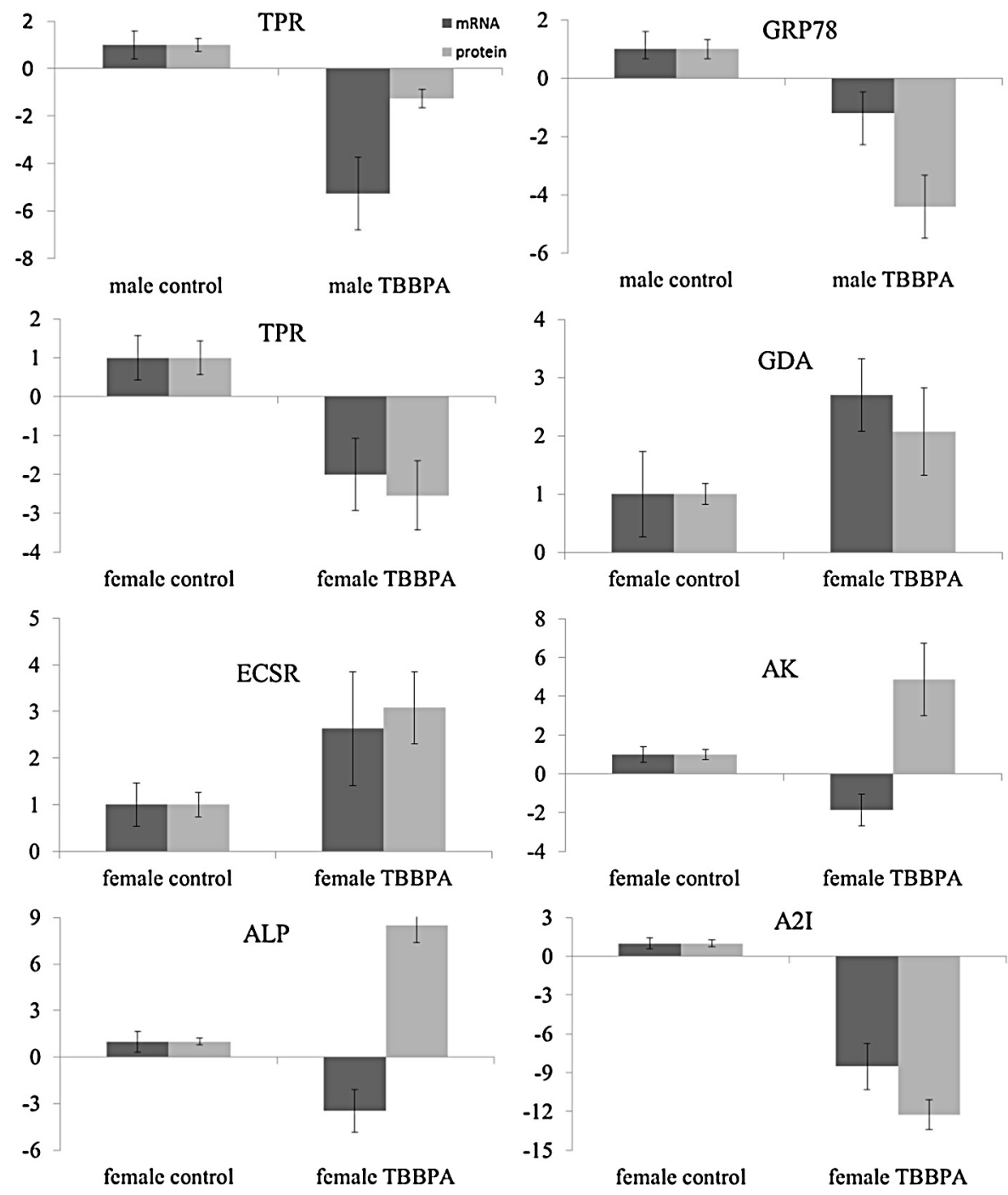

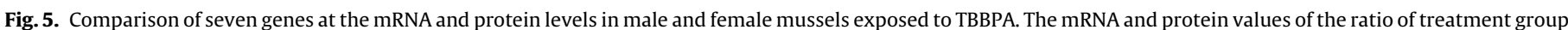

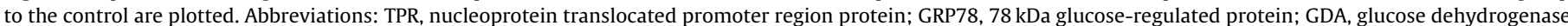
[acceptor]-like; ECSR, epidermal cell surface receptor; AK, arginine kinase; ALP, apextrin-like protein; A2I, apoptosis 2 inhibitor.

\subsection{Differential proteins expressed in male and female mussel hepatopancreas}

The iTRAQ coupled to 2D-LC-MS/MS analysis identified 1043 proteins, of which 31 were of significantly $(p<0.05)$ different abundances in hepatopancreas between male and female mussels. According to the KEGG pathway analysis, these 31 significantly expressed proteins were divided into several pathways including cytoskeleton, reproduction and development, energy and primary metabolism, gene expression, stress response and signal transduction.

Cytoskeleton consisting mostly of microtubules, microfilaments and intermediate filaments plays multiple functions, including cell shape, cell movement, cytokinesis, cell signaling and muscle contraction (Wickstead and Gull, 2011). The higher levels of actin and cytoplasmic dynein combined the lower levels of tropomyosins could imply the differential activity of muscle contraction between male and female mussel samples, while the different levels of suprabasin meant the different processes of epidermal differentiation.

The significant differences of proteins involved directly or indirectly in glucose metabolism implied the metabolic differences between male and female mussels in hepatopancreas. Especially, glyceraldehyde-3-phosphate dehydrogenase and glycosyl hydrolase in male mussel hepatopancreas were more than 13 times higher than those in female mussel hepatopancreas. In addition, arginine kinase and low-density lipoprotein receptor-related protein, related to energy metabolism and chylomicron catabolism respectively (Alonso et al., 2011; Beisiegel et al., 1991), also exhibited differential expression levels between female and male mussel hepatopancreas.

Three differentially expressed proteins including calponin-like protein, epidermal cell surface receptor (ESCR) and sarcoplasmic calcium-binding protein combined with three ribosomal proteins implied the differences in the cellular processes of transduction and translation between male and female mussel samples. Moreover, differential stress-responsive proteins such as apextrin, chitotriosidases glutathione S-transferases (GSTs) and glucose regulated protein (GRP78) suggested that male and female mussel might have different responsive mechanisms to stressors.

Overall, these proteins with different abundances clearly indicated the significant biological differences at protein level between male and female mussel samples. Therefore, there might be differential responses and mechanisms to TBBPA exposure in mussels due to the significant biological differences. 
4.3. Gender-specific proteins responded to TBBPA in male and female mussel hepatopancreas

Among the 60 proteins responded to TBBPA exposure, only two of them (3.3\%) were commonly changed in expression from both male and female mussel samples exposed to TBBPA, which clearly indicated the differential proteomic responses towards TBBPA treatment between male and female mussel samples. These proteins were classified into several groups according to the KEGG database.

\subsubsection{Cytoskeleton}

In TBBPA-treated male mussel hepatopancreas, both actin and tubulin were significantly down-regulated. The similar alteration of actin and tubulin has been also observed in response to cellular stress and apoptosis in M. galloprovincialis exposed to Cylindrospermopsis raciborskii cells (Puerto et al., 2011). Therefore, the alterations of actin and tubulin suggested the cellular stress and apoptosis in TBBPA-treated male mussel samples, which was also evidenced by TUNEL assay. Moreover, suprabasin involved in epidermal differentiation was present in higher concentration in TBBPA-exposed male individuals, suggesting the inhibition of epidermal differentiation by TBBPA exposure. In contrast, the proteomic responses related to cytoskeleton in female mussel samples with TBBPA treatment were completely different with those in male mussel samples. The alterations of septin, catchin and tropomyosin meant the disruption in muscle contraction and subsequent apoptosis induced by TBBPA in female mussel samples, together with the up-regulated cytoplasmic intermediate filament protein.

\subsubsection{Reproduction and development}

Vdg3 and meiosis-specific nuclear structural protein, being of significantly higher expressions in male mussel hepatopancreas as mentioned above, were both significantly up-regulated in female mussel samples, which probably meant that TBBPA induced reproductive toxicities in female mussels, together with the downregulated vitelline envelop receptor for lysin.

\subsubsection{Metabolism}

Ganglioside GM2 activator is a glycolipid transport protein having multiple functions, including innate immunity and lipid metabolism (Klima et al., 1993). Both ganglioside GM2 activator and fatty acid binding protein were down-regulated in male mussel samples after TBBPA exposure, which suggested the disturbance in lipid metabolism induced by TBBPA. The beta subunit of procollagen-proline dioxygenase belongs to the family of protein disulfide isomerases (PDI) catalyzing the formation and breakage of disulfide bonds between sulfides (S-) in cysteine residues of folding proteins. The alterations of procollagen-proline dioxygenase and proteasomes indicated the disturbance in protein metabolism induced by TBBPA. Additionally, the altered $\operatorname{cob}(\mathrm{I})$ yrinic acid a,cdiamide adenosyltransferase, electron transfer flavoprotein (ETF) and ATP synthase demonstrated the disturbance in cobalamin metabolism and energy metabolism in male mussel group with TBBPA exposure (Sheppard et al., 2004; Watmough and Frerman, 2010).

In the female mussel samples treated with TBBPA, glyceraldehyde-3-phosphate dehydrogenase, glucose dehydrogenase (acceptor) and arginine kinase were significantly up-regulated, which suggested that TBBPA mainly disturbed energy metabolisms via metabolic pathways of glucose and arginine in female mussel hepatopancreas. In addition, the up-regulation of glyceraldehyde-3-phosphate dehydrogenase probably implied that female mussels used extra glyceraldehyde3-phosphate dehydrogenase to prevent them from apoptosis and oxidative stress induced by TBBPA exposure (Chandramouli et al., 2013).

\subsubsection{Signal transduction}

In both male and female mussel samples treated with TBBPA, two collagens and one of them were up-regulated with statistical significances, respectively. Collagens are not only essential for the mechanical resistance and resilience of multicellular organisms, but are also signaling molecules defining cellular shape and behavior (Daley et al., 2008). Especially in the female mussel samples exposed to TBBPA, one epidermal cell surface receptor was significantly up-regulated together with collagen alpha-1(XII) chain, which clearly confirmed the disruption in signaling pathways via the extracellular matrix induced by TBBPA. In addition to the alterations of collagens, tyrosine-protein phosphatase non-receptor was down-regulated in male mussel samples treated with TBBPA. Tyrosine-protein phosphatase non-receptor is known to act as a negative regulator of the JAK/STAT signaling pathways downstream of cytokines (Zikherman and Weiss, 2011). Calcium binding proteins are proteins involved in calcium cell signaling pathways by binding to $\mathrm{Ca}^{2+}$ and play important roles in numerous biological processes including muscle contraction, cellular metabolism, cell proliferation, differentiation and apoptosis (Yui et al., 1995). The down-regulation of calcium binding protein probably meant the apoptosis induced by TBBPA in male mussel samples, which was consistent to the alteration of suprabasin.

\subsubsection{Gene expression}

Nucleoprotein TPR, the component of the cytoplasmic fibrils of the nuclear pore complex in nuclear protein import, was reported to be related to apoptosis and oxidative stress (Ferrando-May et al., 2001). In this work, nucleoprotein TPR was significantly down-regulated in both male and female mussel hepatopancreas, indicating the apoptosis induced by TBBPA in mussel samples, as mentioned above. The elongation factors and ribosomal proteins are universal proteins involved in protein biosynthesis occurring in the ribosome, whose proteomic differences suggested the disturbance in protein biosynthesis with differential mechanisms between male and female mussel samples. The up-regulated $\mathrm{ABC}$ excinuclease likely was the indicator of oxidative stress induced by TBBPA in female mussel hepatopancreas, since $A B C$ excinuclease is an ATP-dependent DNA repair enzyme that removes the damaged nucleotides that are often generated by excessive ROS-induced oxidative stress (Orren and Sancar, 1989). In addition, the significant alterations of transcription activator and serine/arginine-rich protein probably meant the disruption in the process of gene expression in female mussels.

\subsubsection{Stress response}

TCTP has multiple functions in diverse organisms and was down-regulated in TBBPA-treated male mussel samples. Recently, evidences have indicated the responses of TCTP to oxidative stresses, such as heavy metals and pathogens, to prevent cell from apoptosis (Li et al., 2010). Heat shock cognate 70 (HSC70) usually functions as a molecular chaperon and exerts important roles in folding of newly synthesized polypeptides, membrane translocation and degradation of misfolded proteins (Gachet et al., 1999). The Hsc70-interacting protein was found to be down-regulated in TBBPA-treated male mussel samples, which probably meant the disruption in protein stability. Similar to Hsc 70 , glucose regulated protein (GRP78) is a stress protein belonging to the $70 \mathrm{kDa}$ heat shock protein family and was also downregulated in TBBPA-treated male mussel samples. Peroxiredoxin, an antioxidant enzyme reducing hydrogen peroxide $\left(\mathrm{H}_{2} \mathrm{O}_{2}\right)$ and alkyl hydroperoxides treatments, regulates peroxide-mediated signaling cascades, while superoxide dismutase catalyzes the 
dismutation of superoxide into oxygen and hydrogen peroxide. Therefore, these altered proteins could be reflected in stronger oxidative stress conditions in the hepatopancreas of male mussels treated with TBBPA than in female mussel samples. In addition, the alterations of selenium binding protein, calreticulin and other stress-response proteins including acidic mammalian chitinase, chitotriosidase and lectin suggested that TBBPA also induced immune responses in male mussel hepatopancreas.

In female mussel hepatopancreas samples, the expressions of lysozyme, apextrin-like protein and chitotriosidase were increased in response to TBBPA exposure. Lysozymes are antibacterial enzymes in the immune system by cleaving the $\beta$ - $(1,4)$-glycosidic bond between $\mathrm{N}$-acetylmuramic acid and $\mathrm{N}$-acetylglucosamine in peptidoglycan layer of bacterial cell walls. Our results demonstrated that the goose-type lysozyme played an important role in digestion indicated by the significantly increased expression level after bacterial challenges (Wang et al., 2012). Apextrin has an important function in neutralization of pathogens and is immunologically responsive to pathogens (Dheilly et al., 2011). Therefore, the significant up-regulation of lysozyme, apextrin-like protein and chitotriosidase clearly indicated the immune responses induced by TBBPA in female mussels.

Clearly, these differential proteins suggested that TBВРA induced oxidative and immune stresses in both male and female mussel hepatopancreas via different metabolic pathways, together with other altered proteins such as $A B C$ excinuclease and glyceraldehyde-3-phosphate dehydrogenase.

\subsubsection{Apoptosis}

In the TBBPA-treated female mussel samples, however, both apoptosis 2 inhibitor (A2I) and cell death protein (CDP) were remarkably down-regulated ( $>8.0$ folds). As members of the antiapoptotic family of proteins, apoptosis inhibitors can inhibit the downstream components of the caspase activation pathways in the regulation of apoptosis in many species (Wei et al., 2008). Cell death proteins play important roles in apoptosis and can be up-regulated during cell death responses in various tissues and after various toxic stress signals (Lankat-Buttgereit and Göke, 2003). Some confirmatory data suggest that cell death proteins act as molecular chaperones to scavenge denatured proteins outside cells following specific stress-induced cellular injuries. The down-regulation of apoptosis inhibitor and cell death protein likely implied that TBBPA could inhibit the biosynthesis of these two proteins and therefore apoptosis occurred in female mussel hepatopancreas after TBBPA exposure for one month.

\subsection{Correlation between gene and protein expressions}

To further evaluate the correlation between gene expression and protein abundances, the expressions of seven genes corresponding to TPR, GRP78, GDA, ECSR, AK, ALP and A2I in M. galloprovincialis were quantified to explore the correlation between protein and mRNA expression levels. Results indicated that the mRNA expressions of both AK and ALP did not correlate with protein expressions. The disparity between mRNA and corresponding protein expressions was not surprising (Wang et al., 2010), since mRNA expression means the tendency of the corresponding encoded protein which does not always happen due to the posttranscriptional and posttranslational modifications (Wang et al., 2010).

\section{Conclusion}

In this study, we used iTRAQ coupled with 2D LC-MS/MS proteomic analysis to investigate the gender-specific responses in mussel Mytilus galloprovincialis exposed to tetrabromobisphenol
A (TBBPA). We confirmed the proteomic differences in hepatopancreas between male and female mussels indicated by 31 differentially expressed proteins. The further proteomic analysis revealed the gender-specific responses between male and female mussel samples to TBBPA exposure, as shown by a total of 60 altered proteins. In details, results indicated that TBBPA exposure could induce apoptosis, immune and oxidative stress and disruption in energy metabolisms in both male and female mussels via different metabolic pathways. In addition, TBBPA exposure could induce disturbances in cell developmental process, lipid and protein metabolisms in male mussel hepatopancreas. In female mussel hepatopancreas, differentially, TBBPA induced reproductive toxicities and disruption in muscle contraction. This work suggested that the gender differences should be considered in ecotoxicoproteomics.

\section{Supporting Information}

The location of the Laizhou Bay in China (Fig. S1), the histological results of gonad for sex determination (Fig. S2), the primer sequences for the determination of housekeeping genes (Table S1), and primer sequences of selected genes for qRT-PCR (Table S2).

\section{Acknowledgments}

This work was supported by National Key Basic Research Program of China (2015CB453303).

\section{Appendix A. Supplementary data}

Supplementary data associated with this article can be found, in the online version, at http://dx.doi.org/10.1016/j.aquatox.2014. 09.008 .

\section{References}

Ahsan, N., Renaut, J., Komatsu, S., 2009. Recent developments in the application of proteomics to the analysis of plant responses to heavy metals. Proteomics 9, 2602-2621.

Alonso, G.D., Pereira, C.A., Remedi, M.S., Paveto, M.C., Cochella, L., Ivaldi, M.S., Gerez de Burgos, N.M., Torres, H.N., Flawiá, M.M., 2011. Arginine kinase of the flagellate protozoa Trypanosoma cruzi. Regulation of its expression and catalytic activity. FEBS Lett. 498, 22-25

Anderson, N.L., Anderson, N.G., 1998. Proteome and proteomics: new technologies, new concepts, and new words. Electrophoresis 19, 1853-1861.

Auffert, M., 1988. Histopathological changes related to chemical contamination in Mytilus edulis from field and experimental conditions. Mar. Ecol. Prog. Ser. 46, 101-107.

Beisiegel, U., Weber, W., Bengtsson-olivecrona, G., 1991. Lipoprotein lipase enhances the binding of chylomicrons to low density lipoprotein receptor-related protein. Proc. Natl. Acad. Sci. 88, 8342-8346.

Birnbaum, L.S., Staskal, D.F., 2004. Brominated flame retardants: cause for concern? Environ. Health Perspect. 112, 9-17.

Bradford, M., 1976. A rapid and sensitive method for the quantification of microgram quantities of protein utilizing the principle of protein-dye binding. Anal. Biochem. 72, 248-254

Cappello, T., Maisano, M., D’Agata, A., Natalotto, A., Mauceri, A., Fasulo, S., 2013a Effects of environmental pollution in caged mussels (Mytilus galloprovincialis). Mar. Environ. Res. 91, 52-60.

Cappello, T., Mauceri, A., Corsaro, C., Maisano, M., Parrino, V., Lo Paro, G., Messina, G., Fasulo, S., 2013b. Impact of environmental pollution on caged mussels Mytilus galloprovincialis using NMR-based metabolomics. Mar. Pollut. Bull. 77, 132-139.

Chandramouli, K.H., Ravasi, T., Reish, D., Qian, x.P.-Y., 2013. Proteomic changes between male and female worms of the polychaetous Annelid Neanthes arenaceodentata before and after spawning. PLoS One 8, e072990.

Ciacci, C., Barmo, C., Gallo, G., Maisano, M., Cappello, T., D’Agata, A., Leonzio, C., Mauceri, A., Fasulo, S., Canesi, L., 2012. Effects of sublethal, environmentally relevant concentrations of hexavalent chromium in the gills of Mytilus galloprovincialis. Aquat. Toxicol. 120-121, 109-118.

Daley, W.P., Peters, S.B., Larsen, M., 2008. Extracellular matrix dynamics in development and regenerative medicine. J. Cell Sci. 121, 255-264.

de Wit, C.A., 2002. An overview of brominated flame retardants in the environment. Chemosphere 46, 583-624.

Dheilly, N.M., Haynes, P.A., Bove, U., Nair, S.V., Raftos, D.A., 2011. Comparative proteomic analysis of a sea urchin (Heliocidaris erythrogramma) antibacterial 
response revealed the involvement of apextrin and calreticulin. J. Invertebr. Pathol. 106, 223-229.

Du, J., Zhu, H., Liu, P., Chen, J., Xiu, Y., Yao, W., Wu, T., Ren, Q., Meng, Q., Gu, W., Wang, W., 2013. Immune response and gene expression in hepatopancreas from Macrobrachium rosenbergii challenged by a novel pathogen spiroplasma MR1008. Fish Shellfish Immunol. 34, 315-323.

Fasulo, S., Iacono, F., Cappello, T., Corsaro, C., Maisano, M., D’Agata, A., De Domenico, E., Parrino, V., Lo Paro, G., Mauceri, A., 2012. Metabolomic investigation of Mytilus galloprovincialis (Lamarck 1819) caged in aquatic environments. Ecotoxicol. Environ. Saf. 84, 139-146.

Ferrando-May, E., Cordes, V., Biller-Ckovric, I., Mirkovic, J., Görlich, D., Nicotera, P., 2001. Caspases mediate nucleoporin cleavage, but not early redistribution of nuclear transport factors and modulation of nuclear permeability in apoptosis. Cell Death Differ. 8, 495-505.

Gachet, Y., Tournier, S., Lee, M., Lazaris-Karatzas, A., Poulton, T., Bommer, U.A., 1999. The growth-related, translationally controlled protein P23 has properties of a tubulin binding protein and associates transiently with microtubules during the cell cycle. J. Cell Sci. 112, 1257-1271.

Garmendia, L., Soto, M., Vicario, U., Kim, Y., Cajaraville, M., Marigomez, I., 2011. Application of a battery of biomarkers in mussel digestive gland to assess longterm effects of the Prestige oil spill in Galicia and Bay of Biscay: tissue-level biomarkers and histopathology. J. Environ. Monit. 13, 915-932.

Han, Z., Sun, J., Zhang, Y., He, F., Xu, Y., Matsumura, K., He, L., Qiu, J., Qi, S., Qian, P., 2013. iTRAQ-based proteomic profiling of the barnacle Balanus amphitrite in response to the antifouling compound meleagrin. J. Proteome Res. 12, 2090-2100

Huang, G., Ying, G., Liang, Y., Zhao, J., Yang, B., Liu, S., Liu, Y., 2013. Hormonal effects of tetrabromobisphenol A using a combination of in vitro and in vivo assays. Comp. Biochem. Physiol. C 157, 344-351.

Ji, C., Wu, H., Wei, L., Zhao, J., Yu, J., 2013. Proteomic and metabolomic analysis reveal gender-specific responses of mussel Mytilus galloprovincialis to 2,2',4,4'tetrabromodiphenyl ether (BDE 47). Aquat. Toxicol. 140-141, 449-457.

Johnson-Restrepoa, B., Adamsb, D.H., Kannan, K., 2008. Tetrabromobisphenol A (TBBPA) and hexabromocyclododecanes (HBCDs) in tissues of humans, dolphins, and sharks from the United States. Chemosphere 70, 1935-1944.

Klima, H., Klein, A., van Echten, G., Schwarzmann, G., Suzuki, K., Sandhoff, K., 1993. Over-expression of a functionally active human GM2-activator protein in Escherichia coli. Biochem. J. 292, 571-576.

Lankat-Buttgereit, B., Göke, R., 2003. Programmed cell death protein 4 (pdcd4): a novel target for antineoplastic therapy? Biol. Cell 95, 515-519.

Li, C., Qiu, L., Ning, X., Chen, A., Qin, S., Wu, H., Zhao, J., 2010. The first molluscan TCTP in Venerupis philippinarum: molecular cloning and expression analysis. Fish Shellfish Immunol. 29, 530-533.

Martyniuk, C.J., Alvarez, S., Denslow, N.D., 2012. DIGE and iTRAQ as biomarker discovery tools in aquatic toxicology. Ecotoxicol. Environ. Saf. 76, 3-10.

Meerts, I.A.T.M., van Zanden, J.J., Luijks, E.A.C., van Leeuwen-Bol, I., Marsh, G., Jakobsson, E., Bergman, A., Brouwer, A., 2000. Potent competitive interactions of some brominated flame retardant and related compounds with human tranthyretin in vitro. Toxicol. Sci. 56, 95-104.

Minguez, L., Brule, N., Sohm, B., Devin, S., Giamberini, L., 2013. Involvement of apoptosis in host-parasite interactions in the zebra mussel. PLoS One 8, e65822.

Moore, M., 1985. Cellular responses to pollutants. Mar. Pollut. Bull. 16, 134-139.

Morris, S., Allchin, C.R., Zegers, B.N., Haftka, J.J.H., Boon, J.P., Belpaire, C., Leonards, P.E.G., van Leeuwen, S.P.J., de Boer, J., 2004. Distribution and fate of HBCD and
TBBPA brominated flame retardants in North Sea estuaries and aquatic food webs. Environ. Sci. Technol. 38, 5497-5504.

Orren, D.K., Sancar, A., 1989. The (A)BC excinuclease of Escherichia coli has only the UvrB and UvrC subunits in the incision complex. Proc. Natl. Acad. Sci. 86 5237-5241.

Pan, X., Tang, J., Li, J., Guo, Z., Zhang, G., 2010. Levels and distributions of PBDEs and PCBs in sediments of the Bohai Sea, North China. J. Environ. Monit. 12, 1234-1241.

Puerto, M., Campos, A., Prieto, A., Cameán, A., de Almeida, A.M., Coelho, A.V., Vasconcelos, V., 2011. Differential protein expression in two bivalve species: Mytilus galloprovincialis and Corbicula fluminea: exposed to Cylindrospermopsis raciborskii cells. Aquat. Toxicol. 101, 109-116.

Pütz, Stephanie, M., Boehm, A.M., Stiewe, T., Sickmann, A., 2012. iTRAQ analysis of a cell culture model for malignant transformation, including comparison with 2D-PAGE and SILAC. J. Proteome Res. 11, 2140-2153.

Sheppard, D.E., Penrod, J.T., Bobik, T., Kofoid, E., Roth, J.R., 2004. Evidence that a B12 adenosyl transferase is encoded within the ethanolamine operon of Salmonella enterica. J. Bacteriol. 186, 7635-7644.

Shilov, I.V., Seymour, S.L., Patel, A.A., Loboda, A., Tang, W.H., Keating, S.P., Hunter, C.L. Nuwaysir, L.M., Schaeffer, D.A., 2007. The paragon algorithm, a next generation search engine that uses sequence temperature values and feature probabilities to identify peptides from tandem mass spectra. Mol. Cell. Proteomics 6 1638-1655.

Strack, S., Detzel, T., Wahl, M., Kuch, B., Krug, H.F., 2007. Cytotoxicity of TBBPA and effects on proliferation, cell cycle and MAPK pathways in mammalian cells. Chemosphere 67, S405-S411.

Szymanska, J.A., Piotrowski, J.K., Frydrych, B., 1999. Hepatotoxicity of tetrabromobisphenol-A: effects of repeated dosage in rats. Toxicology 142, 87-95.

Wang, Q., Zhang, L., Zhao, J., You, L., Wu, H., 2012. Two goose-type lysozymes in Mytilus galloprovincialis: possible function diversification and adaptive evolution. PLoS One 7, e45148.

Wang, X., Chang, L., Sun, Z., Zhang, Y., 2010. Comparative proteomic analysis of differentially expressed proteins in the earthworm Eisenia fetida during Escherichia coli 0157:H7 stress. J. Proteome Res. 9, 6547-6560.

Watmough, N.J., Frerman, F.E., 2010. The electron transfer flavoprotein: Ubiquinone oxidoreductases. Biochim. Biophys. Acta 1797, 1910-1916.

Weckwerth, W., 2011. Green systems biology - From single genomes, proteomes and metabolomes to ecosystems research and biotechnology. J. Proteomics 75, 284-305.

Wickstead, B., Gull, K., 2011. The evolution of the cytoskeleton. J. Cell. Biol. 194 513-525.

Wei, Y., Fan, T., Yu, M., 2008. Inhibitor of apoptosis proteins and apoptosis. Inhibitor of apoptosis. Acta Biochim. Biophys. Sin. 40, 278-288.

Yui, S., Mikami, M., Yamazaki, M., 1995. Induction of apoptotic cell death in mouse lymphoma and human leukemia cell lines by a calcium-binding protein complex, calprotectin, derived from inflammatory peritoneal exudate cells. J. Leukoc. Biol. 6, 650-658.

Zhang, L., Yun, X., Na, G., Chen, T., Zhang, Y., Gu, J., Liu, C., 2011. Determination and application of TBBPA in water by high performance liquid chromatographytandem mass spectrometry. Chin. J. Environ. Eng. 5, 1077-1080.

Zikherman, J., Weiss, A., 2011. Unraveling the functional implications of GWAS: how $\mathrm{T}$ cell protein tyrosine phosphatase drives autoimmune disease. J. Clin. Invest. $121,4618-4621$. 